\title{
Surgical video on the successful preservation of the non-recurrent laryngeal nerve and the importance of preoperative CT imaging during total thyroidectomy: case report
}

\author{
Allen Young^^, Robert C. Wang \\ Department of Otolaryngology Head and Neck Surgery, University of Nevada Las Vegas, Las Vegas, NV, USA \\ Correspondence to: Allen Young, MD, MPH. University of Nevada Las Vegas, 1701 West Charleston Boulevard Suite 490, Las Vegas, NV 89102, USA. \\ Email: AllenHYoung@gmail.com.
}

\begin{abstract}
This presentation demonstrates the exposure and preservation of a non-recurrent laryngeal nerve (NRLN) during a total thyroidectomy for a large thyroid goiter using a video for the first time in the literature, and reinforces the importance of preoperative imaging to detect an aberrant right subclavian artery to suspect the presence of a NRLN. A NRLN is a rare anatomic variation that presents a unique technical challenge during thyroid and parathyroid surgery. An undetected NRLN has a 6-fold increase in intraoperative nerve injuries, resulting in hoarseness, aspiration, and decreased quality of life. A 29-yearold female had a large thyroid goiter with compressive symptoms and a preoperative CT scan with contrast showing a retro-esophageal right subclavian artery. Biopsy showed benign thyroid tissue and she underwent a total thyroidectomy with intraoperative nerve monitoring (IONM). During surgery, a right NRLN candidate was seen coursing towards the laryngotracheal junction (LTJ) from laterally and superiorly, rather than from inferiorly. Dissection in the inferior-paratracheal area revealed the vagus nerve laterally, but no inferior recurrent laryngeal nerve. The nerve was traced to its entrance into the larynx, confirming a NRLN. The thyroid goiter was removed with preservation of the parathyroid glands, right NRLN, and left recurrent laryngeal nerve. The patient had normal postoperative vocal cord motion and parathyroid function. Surgeons can reduce the risk of intraoperative nerve damage through preoperative CT imaging to rule out aberrant subclavian artery, awareness of the different anatomical pathways of the NRLN, and IONM.
\end{abstract}

Keywords: Non-recurrent laryngeal nerve (NRLN); aberrant subclavian artery; goiter; thyroidectomy; case report

Received: 04 July 2020; Accepted: 17 December 2020; Published: 20 April 2022.

doi: 10.21037/jovs-20-144

View this article at: http://dx.doi.org/10.21037/jovs-20-144

\section{Introduction}

Iatrogenic damage to a unilateral recurrent laryngeal nerve (RLN) during thyroid and parathyroid surgery can result in dysphonia, dysphagia, aspiration, and in bilateral RLN injury, airway compromise requiring a tracheostomy (1). Non-recurrent laryngeal nerve (NRLN) is a rare anatomic variation, caused by an aberrant embryological origin of the subclavian artery, that presents an unusual surgical challenge with high rates of intraoperative nerve injuries, if unsuspected and undetected (2). The incidence of nonRLN varies across different studies from $0.4 \%$ to $4.76 \%$ $(2,3)$. During preoperative planning, it is important to assess for a retro-esophageal right subclavian artery on CT imaging with contrast and be aware of its association with a NRLN. Intraoperatively, knowledge of the different

^ORCID: 0000-0002-3871-6122. 


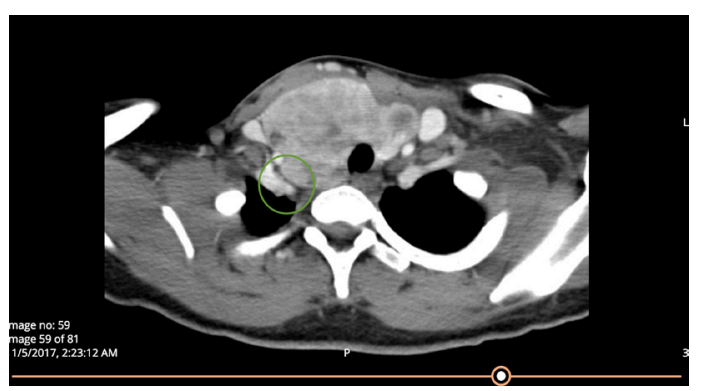

Figure 1 Preoperative CT neck showed a $7.7 \times 4.5 \times 3.6 \mathrm{~cm}^{3}$ right thyroid lobe, a $7.1 \times 3.3 \times 3.1 \mathrm{~cm}^{3}$ left lobe, and a retro-esophageal, right subclavian artery (circled in green). The right lobe extends into the paraoesophageal area abutting the prevertebral fascia.

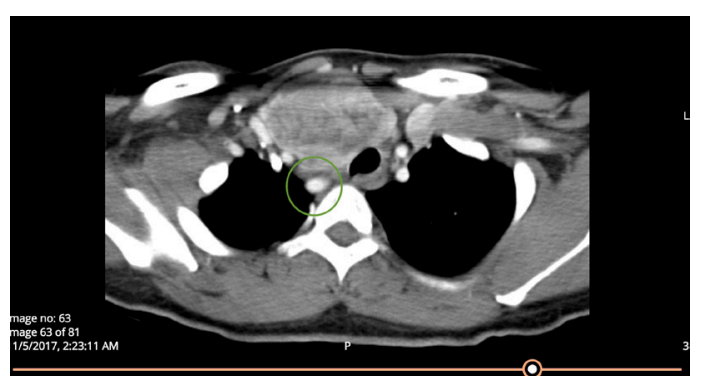

Figure 2 Retro-esophageal, right subclavian artery (circled in green) crossing the midline.

anatomic courses of a NRLN and appropriate dissection to isolate the NRLN are important to prevent nerve damage and vocal paralysis. To the best of our knowledge, this is the first video presentation that shows preoperative CT imaging of an aberrant right subclavian artery and the subsequent identification and successfully preservation of a NRLN during a total thyroidectomy for a large thyroid goiter. We present the following article in accordance with the CARE reporting checklist (available at https://jovs.amegroups. com/article/view/10.21037/jovs-20-144/rc).

\section{Case presentation}

All procedures performed in this study were in accordance with the ethical standards of the institutional research committee and with the Helsinki Declaration (as revised in 2013). Written informed consent was obtained from the patient for publication of this case report and accompanying images. A copy of the written consent is available for review by the editorial office of this journal.
A 29-year-old female presented with a history of an enlarging, non-tender thyroid gland for 3 years. She complained of dysphagia, orthopnea, and fatigue, but denied any voice changes. Fine-needle aspiration showed benign thyroid tissue and her thyroid labs were within normal limits. Her preoperative CT scan of the neck with contrast showed a $7.7 \times 4.5 \times 3.6 \mathrm{~cm}^{3}$ right thyroid lobe, a $7.1 \times 3.3 \times 3.1 \mathrm{~cm}^{3}$ left lobe, and a retro-esophageal, right subclavian artery (Figures 1,2). She agreed to proceed to total thyroidectomy after discussion and informed consent.

A flowchart of our surgical technique is shown in Figure 3. The patient was placed supine on the operating table with the neck in extension. Intraoperative nerve monitoring (IONM) was performed using an EMG electrode-equipped endotracheal tube. A $6 \mathrm{~cm}$ horizontal neck incision was made through an existing skin crease using monopolar cautery down to the level of the platysma. Subcutaneous flaps were elevated superiorly to the hyoid bone and inferior to the sternal notch and retraction held using a Lone Star Self-Retaining Retractor. The strap muscles were divided along the median raphe and elevated off the surface of the thyroid gland using a Harmonic Scalpel and the right sternothyroid muscle was transected to allow access to the large right-sided goiter (Video 1, 0:08-0:13). The isthmus was transected to expose the underlying trachea and the smaller left thyroid lobe approached first (Video 1, $0: 14-0: 18)$. The inferior and superior medial aspect of Berry's ligament was transected and the left superior thyroid vessels ligated on the surface of the thyroid gland (Video 1, $0: 30-0: 35)$. The thyroid gland was retracted superiorly and medially, exposing the deep surface of the inferior thyroid. The left recurrent laryngeal nerve was located and traced superiorly (Video 1, 0:19-0:29). The soft tissues containing the superior and inferior parathyroid glands with their vasculature were dissected away from the left thyroid lobe. The remaining Berry's ligament was then transected medial to the recurrent laryngeal nerve and the left thyroid lobe delivered off the field (Video 1, 0:36-0:43).

The larger right thyroid lobe, noted by preoperative imaging to extend to the paraesophageal area and prevertebral fascia (Figure 1), was exposed and the right inferior and superior medial Berry's ligament divided. The thyroid lobe was reflected superiorly and the middle thyroid vein and inferior thyroid vessels ligated on the lateral and deep surface of the inferior right thyroid gland, reflecting the inferior parathyroid gland and its blood supply away from the thyroid lobe. The recurrent laryngeal nerve was not identified at its usual position at the tracheoesophageal 


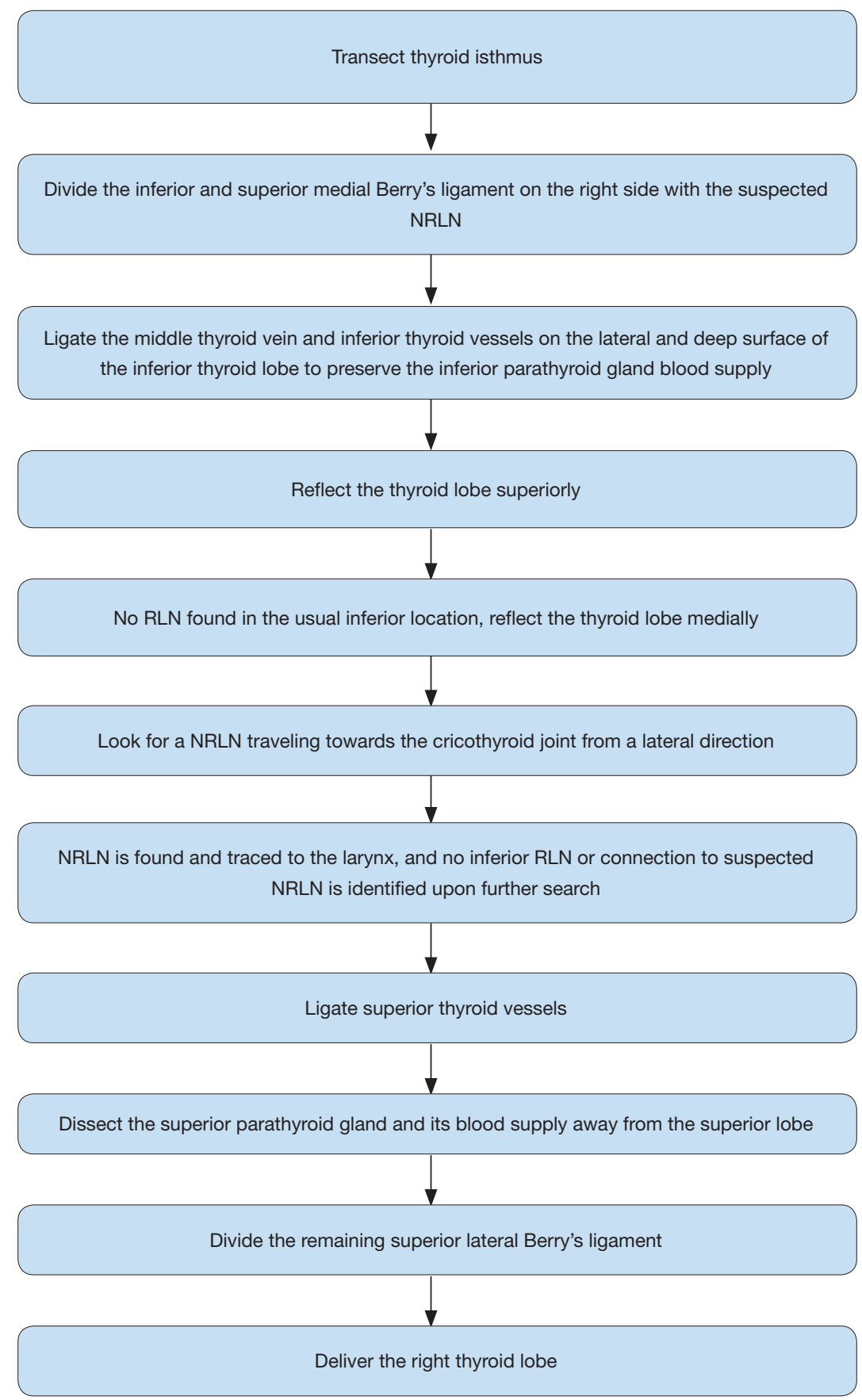

Figure 3 Flowchart of surgical steps for preserving the non-recurrent laryngeal nerve during total thyroidectomy. NRLN, non-recurrent laryngeal nerve; RLN, recurrent laryngeal nerve; LTJ, laryngotracheal junction.

groove. Given the preoperative finding of an aberrant right subclavian artery, our suspicion for a NRLN was high. The thyroid gland was reflected medially and a nerve was discovered traveling toward the inferior posterior aspect of the LTJ from a lateral and superior course (Video 1, 1:50-
2:19). A visual illustration of an example NRLN course by Henry et al. (3) is shown in Video 1, 2:18-2:19.

Further dissection in the right inferior paratracheal area revealed the vagus nerve laterally without any suggestion of an inferior recurrent laryngeal nerve or connection to 


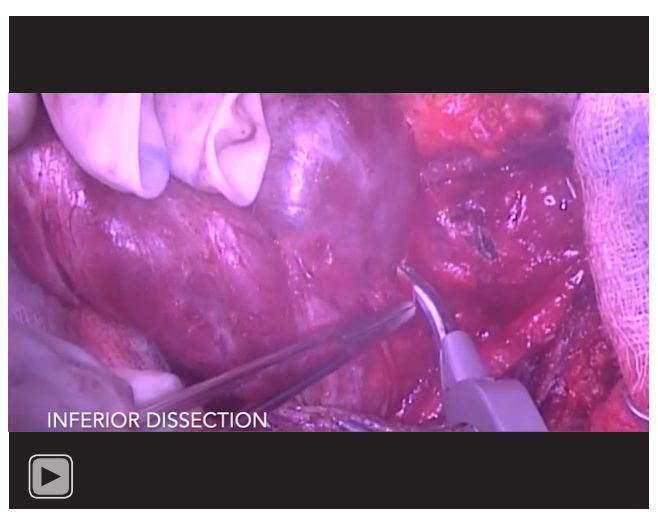

Video 1 Total thyroidectomy of a large thyroid goiter.

the previously dissected NRLN candidate. The NRLN candidate nerve was dissected along its course to its entry into the larynx (Video 1, 2:21-2:40). The right superior thyroid vessels were ligated on the surface of the thyroid gland with care not to injure the external branch of the superior laryngeal nerve. The soft tissues containing the right superior parathyroid gland with its vasculature were dissected away from the thyroid lobe (Video 1, 2:41-2:51). The remainder of Berry's ligament was divided superiorly and laterally, and the right lobe was delivered off the field (Video 1, 2:53-3:20).

Following hemostasis, the strap muscles were reapposed in the midline as were the subcutaneous adipose tissue and dermal layers using 4-0 Vicryl. Dermabond was used to appose the skin edges, and no drain was used. The patient tolerated the procedure well and was discharged on postoperative day one with a normal voice, airway, calcium and parathyroid hormone levels. Final pathology revealed adenomatous hyperplasia without malignancy. At one week's follow-up, she had normal bilateral vocal cord motion on flexible laryngoscopy and normal calcium and parathyroid hormone levels.

\section{Discussion}

The NRLN is the result of abnormal branchial arch development. Embryologically, both RLN supply the $6^{\text {th }}$ branchial arch and pass beneath the $4^{\text {th }}$ branchial arch, which becomes the aortic arch on the left and the subclavian artery on the right. During development, these arteries descend into the chest, bringing along the RLN and creating their elongated paths. However, when the right $4^{\text {th }}$ branchial arch is obliterated or regresses, the right subclavian artery becomes anomalous and originates from the left aortic arch (arteria lusoria) (4). The aberrant subclavian artery then crosses the midline behind the esophagus (which can produce dysphagia lusoria) and allows the inferior laryngeal nerve to be non-recurrent from the vagus nerve $(4,5)$. This NRLN takes a direct path medially to the right larynx (2). Due to its embryological development, NRLN mainly occurs on the right side. However, left sided NRLN can occur in cases of situs inversus combined with an aberrant left subclavian artery and right-sided ligamentum arteriosum with an incidence of $0.04 \%$ (6).

The course of a NRLN may vary with the origin from the vagus lying above the laryngotracheal junction (LTJ) in $58.3 \%$ and below in $41.7 \%$ (3). Cagnol et al. (7) describes two path types. In Type 1 anomaly, NRLN originates more superiorly from the vagus and descends to the larynx, running together with vessels of superior thyroid lobe, creating a higher risk of nerve injury when tying off the superior thyroid vessels $(7,8)$. In Type 2 , NRLN originates from the vagus more inferiorly and follows a transverse path parallel to the inferior thyroid artery (7). Abboud et al. (9) reported 3 types: Type Ia (straight, transverse course from the level of upper thyroid lobe), type Ib (most common, straight transverse course from the level of thyroid isthmus), and type II (initial downwards curve, then runs upwards to the lower pole of the thyroid gland). Hong et al. (2) describes 4 types: descending type (NRLN descends after originating from vagus), vertical type (NRLN runs straight to the LTJ), ascending type (NRLN runs upward to the LTJ), and V-shaped type (NRLN takes initial downward direction and runs upward to LTJ). Our patient had the NRLN originate above the LTJ and descend towards the larynx, consistent with a Type 1 according to Cagnol et al. (7), type 1a according to Abbound et al. (9), and descending type according to Hong et al. (2). From a surgical perspective, it is vital to isolate the entire course of the NRLN prior to transecting the remaining Berry's ligament to free the thyroid lobe, due to the risk of injuring NRLN taking a looping course as seen in Type II (9) or a V-shaped type (2) NRLN.

There is a 6 -fold increase in intraoperative nerve injures when patients have an undetected NRLN. Most patients with NRLN who experienced injuries had nerves originating above the LTJ and coursing with the superior thyroid artery $(8,10)$. A NRLN is almost always associated with an aberrant subclavian artery (2). Preoperative identification of an aberrant right subclavian artery is the best defense against NRLN nerve injury for a surgeon. Symptoms of aberrant subclavian are often silent, but 
if present, can include dysphagia, chronic cough, and unexplained ischemia of right upper limb (11). CT imaging with contrast can provide preoperative surgical planning and can identify an aberrant subclavian artery $100 \%$ of the time $(5,12)$. In addition, intraoperative EMG monitoring can be used to help isolate and preserve unusual variations of the RLN (13).

\section{Patient perspective}

On postoperative follow up, the patient reports resolution of prior dysphagia, orthopnea, and fatigue. She states she is now able to sleep comfortably without any compressive symptoms over her neck. Furthermore, with the resolution of her bulky neck appearance, she is no longer embarrassed to attend public events. She was pleased with the decision to surgically remove her thyroid.

\section{Conclusions}

The risk of causing vocal cord palsy is significantly increased in performing thyroidectomy in patients with NRLN. Surgeons can reduce the risk of NRLN damage through preoperative CT imaging to assess for an aberrant subclavian artery, awareness of the different anatomical pathways of the NRLN, and IONM.

\section{Acknowledgments}

This study was presented at the American Academy of Otolaryngology Head and Neck Surgery (AAO-HNS) 2019 Annual Meeting in September 2019 in New Orleans, LA, USA.

Funding: None.

\section{Footnote}

Reporting Checklist: The authors have completed the CARE reporting checklist. Available at https://jovs.amegroups. com/article/view/10.21037/jovs-20-144/rc

Conflicts of Interest: Both authors have completed the ICMJE uniform disclosure form (available at https://jovs. amegroups.com/article/view/10.21037/jovs-20-144/coif). The authors have no conflicts of interest to declare.

Ethical Statement: The authors are accountable for all aspects of the work in ensuring that questions related to the accuracy or integrity of any part of the work are appropriately investigated and resolved. All procedures performed in this study were in accordance with the ethical standards of the institutional research committee and with the Helsinki Declaration (as revised in 2013). Written informed consent was obtained from the patient for publication of this case report and accompanying images. A copy of the written consent is available for review by the editorial office of this journal.

Open Access Statement: This is an Open Access article distributed in accordance with the Creative Commons Attribution-NonCommercial-NoDerivs 4.0 International License (CC BY-NC-ND 4.0), which permits the noncommercial replication and distribution of the article with the strict proviso that no changes or edits are made and the original work is properly cited (including links to both the formal publication through the relevant DOI and the license). See: https://creativecommons.org/licenses/by-nc-nd/4.0/.

\section{References}

1. Yu WV, Wu CW. Speech therapy after thyroidectomy. Gland Surg 2017;6:501-9.

2. Hong KH, Park HT, Yang YS. Characteristic travelling patterns of non-recurrent laryngeal nerves. J Laryngol Otol 2014;128:534-9.

3. Henry BM, Sanna S, Graves MJ, et al. The NonRecurrent Laryngeal Nerve: a meta-analysis and clinical considerations. PeerJ 2017;5:e3012.

4. Wang $\mathrm{Y}, \mathrm{Ji}$ Q, Li D, et al. Preoperative CT diagnosis of right nonrecurrent inferior laryngeal nerve. Head Neck 2011;33:232-8.

5. Watanabe A, Taniguchi M, Kimura Y, et al. Efficient, effective, safe procedure to identify nonrecurrent inferior laryngeal nerve during thyroid surgery. Head Neck 2016;38:573-7.

6. Jatzko GR, Lisborg PH, Müller MG, et al. Recurrent nerve palsy after thyroid operations--principal nerve identification and a literature review. Surgery 1994;115:139-44.

7. Cagnol G, Santini J, Demard F. Anatomy of the recurrent nerve. Descriptive anatomy, trajectory and relationships, variations. Acta Otorhinolaryngol Belg 1987;41:821-7.

8. Mra Z, Wax MK. Nonrecurrent laryngeal nerves: anatomic considerations during thyroid and parathyroid surgery. Am J Otolaryngol 1999;20:91-5. 
9. Abboud B, Aouad R. Non-recurrent inferior laryngeal nerve in thyroid surgery: report of three cases and review of the literature. J Laryngol Otol 2004;118:139-42.

10. Toniato A, Mazzarotto R, Piotto A, et al. Identification of the nonrecurrent laryngeal nerve during thyroid surgery: 20-year experience. World J Surg 2004;28:659-61.

11. Natsis K, Lazaridis N, Gkiouliava A, et al. Retrooesophageal right subclavian artery in association with thyroid ima artery: a case report, clinical impact

doi: 10.21037/jovs-20-144

Cite this article as: Young A, Wang RC. Surgical video on the successful preservation of the non-recurrent laryngeal nerve and the importance of preoperative CT imaging during total thyroidectomy: case report. J Vis Surg 2022;8:20. and review of the literature. Folia Morphol (Warsz) 2016;75:130-5.

12. Page C, Monet $\mathrm{P}$, Peltier J, et al. Non-recurrent laryngeal nerve related to thyroid surgery: report of three cases. J Laryngol Otol 2008;122:757-61.

13. Barczyński M, Konturek A, Pragacz K, et al. Intraoperative nerve monitoring can reduce prevalence of recurrent laryngeal nerve injury in thyroid reoperations: results of a retrospective cohort study. World J Surg 2014;38:599-606. 